\title{
HOMENAJE A ARLT, BORGES Y ONETTI DE RICARDO PIGLIA
}

\author{
POR \\ RITA GNUTZMANN \\ Universidad del País Vasco, Vitoria
}

En 1975 Ricardo Piglia publica el libro Nombre falso que, pese a haber pasado desapercibido, tiene entre otros el interés de anticipar en varios aspectos su novela Respiración artificial (1980). Los textos de Nombre falso son difíciles de clasificar, a pesar de que se los califique de "cuentos" en la contraportada. E1 único elemento que les une es su carácter enigmático y el tema del fracaso. En el primer texto, "El fin del viaje", se relata el viaje de un periodista literario, Emilio Renzi, a Mar del Plata para ver a su padre ingresado en una clínica tras un intento de suicidio. No cabe duda de que este Renzi es el esbozo del personaje de Respiración artificial, igualmente escritor, quien hace un viaje a Concordia para visitar a su tío Marcelo Maggi que posiblemente acaba de morir y quien, al igual que el padre en el cuento, se preocupó toda su vida de "conservar su propia dignidad" (Respiración, 274). En los cinco años que median entre el cuento y la novela la situación política en Argentina ha cambiado y con ello el enfoque de los dos textos: de una historia individual se pasa a un texto de significado político, a saber, la desaparición de un hombre en la Argentina de la dictadura militar ${ }^{1}$.

Otro rasgo anticipador en el cuento es su insistencia en la relación entre vida y escritura, la transformación de la primera en la segunda mediante el diario que lleva el protagonista. El mismo rasgo se repite en "La caja de vidrio", en la que un narrador en primera persona relata la subyugación y humillación a la que le somete un tal Rinaldi tras un extraño accidente acaecido a un niño. El cuento, a pesar de su forma en primera persona incluye extractos de los manuscritos del enigmático opresor Rinaldi.

Dejo de lado los otros tres textos, "El Laucha Benítez cantaba boleros", "Las actas del juicio" y "El precio del amor", puesto que no interesan en el contexto de este estudio.

\footnotetext{
${ }^{1}$ Cf. la entrevista de Piglia con Marithelma Costa (1986); sobre el aspecto político de la novela véase M. Morello-Frosch (1985) y Rita Gnutzmann (1990).
} 
El texto de mayor extensión es el titulado "Homenaje a Roberto Arlt", dedicado, no por casualidad, a una crítica literaria, Josefina Ludmer. Está subdividido en una primera parte sin título y cuatro partes numeradas (de 1 a 4) a las que sigue un "Apéndice: Luba", es decir, las primeras partes sirven de introducción al cuento arltiano, "Luba". Como objetivo principal se establece desde el comienzo la busca de este cuento y el esclarecimiento de su autoría. El narrador en primera persona, del que sólo en la última parte sabemos que se llama como su autor, Ricardo Piglia (143), presenta su manuscrito como "informe" $\mathrm{e}$ "investigación". La parte introductoria (99-103) parece describir la típica labor de un crítico literario que establece sus fichas y sus documentos de una forma "ordenada y objetiva". En el mejor estilo de Borges, menciona detalles fácilmente comprobables como la muerte de Arit en 1942 y algunos títulos del autor en aquel momento no recogidos en forma de libro, como su ensayo "Las ciencias ocultas en la ciudad de Buenos Aires" (ahora incluido en Nuevas aguafuertes porteñas, 1975 y en Obra completa, 1981), y el capítulo "El poeta parroquial", descartado de su novela El juguete rabioso, y publicado en 1925 en la revista Proa. Este capítulo en efecto se transformó "en la versión final de la novela en el encuentro con Timoteo Souza" (99), dato no reconocido por la mayoría de los críticos y que comprueba el buen conocimiento de la obra arltiana por parte del narrador. Los problemas de esta investigación "fidedigna" comienzan con las cinco aguafuertes "que no habían sido recogidas en libro". Cualquier investigador arltiano sabe que aún falt ta por publicar aproximadamente un $80 \%$ de sus aguafuertes; pero una primera labor de recopilación fue llevada a cabo por D. C. Scroggins en los años 70 . Aunque esta recopilación sea incompleta (faltan los meses desde mayo de 1933 hasta principios de 1935), no he podido encontrar (salvo error mío) tres de las aguafuertes mencionadas por Piglia ("Un perro andaluz", "Genios de Buenos Aires", "Alegría fúnebre"), mientras que las otras dos, pretendidamente inéditas ("La inutilidad de los libros", "La terrible sinceridad") se encuentran entre las que más veces han sido publicadas: la primera en Cronicón de sí mismo (1969) y Aguafuertes porteñas (1973) y la segunda tres veces, en Aguafuertes porteñas (1950), Cronicón y Aguafuertes porteñas (1973). El narrador se confunde igualmente con los datos de la obra dramática, Separación feroz que, según la recopiladora bibliográfica E. E. Rossi, no se publicó el 18 de agosto de 1938 sino el 1 de enero del mismo añoy que además fue publicado en setiembre de 1957 en la revista Punto y Aparte (en D. Guerrero, 196). Vemos que en esta primera parte el "investigador" Piglia se ha equivocado en varios datos, lo que hace dudar de su veracidad ${ }^{2}$.

A continuación ("2. El conjunto de sus escritos inéditos") se presentan:

${ }^{2}$ Dicho sea de paso, también "El poeta parroquial" se volvi6 a publicar en Los libros 29, marzo-abril 1973, tal vez instigado por el propio Piglia, ya que en el mismo número aparece su artículo "Roberto Arlt: una crítica de la economía literaria". 
a. un retrato autobiográfico,

b. notas para una novela en preparación

c. el cuento "Luba".

Es difícil comprobar si es cierto que en 1939 un editor pensaba publicar juntas las novelas Los siete locos y Los lanzallamas, pero resulta sospechoso este editor por llevar el mismo nombre del protagonista del cuento anterior y por su extraño apellido "Moied". Tal vez se trata de una autorreferencia, ya que, leído a la inversa, resulta "de moi". Sigue la investigación erudita, que incluye notas a pie de página, en este caso la reproducción del "retrato autobiográfico de Arlt". Repite lo que ya se sabe de aguafuertes como "Qué nombre le ponemos al pibe" y de autorretratos recogidos por Mirta Arlt y Omar Borré en Para leer a Roberto Arlt (1984). Comienza con el mismo tono arltiano humorístico de sus escritos menores para luego expresar ideas acerca de la literatura como robo, que él mismo nunca expresó de una forma tan coherente, sino que están tomadas de cierta corriente crítica argentina, en la que se encuentra el propio Piglia. Cito como títulos más representativos los dos ensayos del propio Piglia, "Roberto Arlt: una crítica de la economía literaria" (1973) y "Roberto Arlt: la ficción del dinero" (1974); también Mario Goloboff y Noé Jitrik defienden la misma teoría en sus ensayos "La primera novela de Roberto Arlt: el asalto a la literatura" (1975) y "Entre el dinero y el ser" (1976). La literatura como "falsificación burda, de otras falsificaciones que también se inspiraron en falsificaciones" (nota 1, 101) es una evidente interpretación de la obra arltiana por parte de Piglia y resume el tema central de su propio texto "Homenaje a Roberto Arlt" ${ }^{\text {"3. }}$.

El discurso crítico de investigación que ha dominado hasta este momento se transforma repentinamente en discurso ficticio. Introduce a un tal Andrés Martina que alquiló a Arlt un galpón para sus experimentos. Al lector no se le permite la duda sobre la veracidad del personaje, ya que a la manera borgiana, se enlaza con otro, Pascual Nacaratti, documentado por uno de los primeros críticos arltianos, Raúl Larra $(1962,18)$. Si realmente se tratara de un discurso científico el narrador deberfa exponer breve y objetivamente la historia del hallazgo del cuaderno de Arlt y comprobar su autenticidad. En vez de ello pone en boca de su personaje ficticio Martina el relato de su relación con Arlt, de algún accidente de éste y la existencia de "montones de notas sobre una novela", de la que anticipa a la manera detectivesca un breve resumen argumental. Esta parte ficticia, dialogada, termina con la exhortación del detective (Piglia) a su ayudante (Martina) a que no dé ninguna información a nadie sobre el hallazgo, para crear suspenso al final de capítulo, al mejor estilo de las novelas policíacas.

${ }^{3}$ No podría afirmar con seguridad que no existe la recopilación de la Correspondencia mencionada en la nota 2, pero desde luego no la he visto citada en ninguna bibliografía sobre Arlt, es decir, se trata de una publicación apócrifa al estilo de Borges. 
Pretende haber olvidado que lo que en realidad nos había prometido no eran unas notas para una novela sin título sino el cuento "Luba", es decir, usa la técnica de retardamiento típica del relato policíaco.

La parte " 1 " vuelve a la investigación literaria con la descripción de] cuaderno y una transcripción de las notas “arltianas”, interrumpidas por breves resúmenes y notas aclaratorias a pie de página. Hay varios elementos que hacen sospechosa la verdad de la investigación. En primer lugar llama la atención el nombre de Rinaldi, puesto que Arlt casi siempre suele elegir nombres de origen nórdico como Astier, Haffner, Balder, Erdosain ${ }^{4}$, tal como lo practica más tarde otro admirador de Arlt, Juan Carlos Onetti. En Arlt los nombres italianos se suelen limitar a personajes secundarios. Pero es más: en el resumen del cuento "La caja de cristal" vimos que uno de los dos protagonistas se llamaba Rinaldi y el propio narrador ayudaba al lector para descubrir la identidad de los dos personajes: los dos tienen un jadeo asmático que se transcribe literalmente:

¿No gusta (jadeo) tomar (jadeo) una cerveza (jadeo)? (60);

"Qué es - jadeo-robar -jadeo-un banco-jadeo-comparado con - jadeofundarlo" (105).

Curiosamente, en la tercera parte, se introduce a otro personaje con el mismo defecto: Kostia, el ladrón del cuento "Luba", quien además parece ocupar la misma mesa del mismo bar que Rinaldi (129). Una vez alertado ante estas "falsificaciones", el lector se dará cuenta de que el resumen de las notas de la novela coincide prácticamente con el argumento del cuento "La caja de cristal":

En medio de la multitud, en la jungla de la ciudad, imaginar a un hombre cuyo destino y cuya vida están en poder de otro, como si los dos estuvieran en un desierto (104).

Sin embargo, la temática general y el ambiente que el "investigador" resume de las notas arltianas, parecen auténticos: la atracción por la belleza del crimen (como Silvio que se creerá "hermoso como Judas Iscariote" si traiciona al Rengo). La adopción de una niña de ocho años, "Maria, hermosa y renga" hace alusión a varios episodios arltianos: la perversión de la niña perpetrada por Erdosain, relatada en dos ocasiones en las que Arlt, en efecto, se confunde en la edad de la niña (aunque no cambie, como dice Piglia, de 8 a 12 años, sino de 9 a 8, Los siete locos,(227), Los lanzallamas (181) y "Homenaje", 109). Pero lo importante es que mencione un típico elemento de la escritura arltiana: la

\footnotetext{
${ }^{4}$ Es cu rioso cómo los críticos han interpretado el nombre Erdosain; Alain Bosquet sugiere un origen alemán, relacionándolo con "Erde" (tierra), idea retomada por M. Arlt y O. Borré $(1984,71)$. Goloboff sugiere la palabra francesa "sain" (sano) como constituyente del nombre $(1988,74)$ y Vanasco incluso habla de un apellido "raro y siniestro" $(1972,19)$. En realidad se trata de un apellido vasco bastante común.
} 
despreocupación por detalles como la edad de los personajes (igual ocurre con Barsut), dos diferentes casualidades que salvan a Haffner de la muerte etc. El episodio resumido, en el que se entrega a la niña María ("Homenaje" 105) podría referirse, además, a otro de la obra arltiana, a saber, la escena en la que doña Ignacia vende a su hija, la Bizca (la María de Piglia es renga), que igualmente se llama María. El personaje de María es muy discutido por los críticos de Arlt; Vanasco, por ejemplo, piensa que se trata de la transformación del modelo de María Timofoievana Libiadkina (renga como la de Piglia) de Los demonios de Dostoievski ${ }^{5}$. También la alusión a la "conciencia de superioridad" del personaje coincide con los relatos arltianos, igual que su énfasis en ciencias como la geometría, y sobre todo la calificación del protagonista como "un loco" al igual que los famosos de la novela de Arlt. Otros ingredientes típicamente arltianos en la descripción del protagonista Lettif son sus contradicciones ("Profesión de fe religiosa en que se declara ateo"), su intento de suicidio (como Silvio, Haffner, el Astrólogo, Hipólita, Erdosain ...), sus tratados científicos (como los croquis y fórmulas en Los lanzallamas $)^{6}$, la misoginia que profesa, etc.

También esta parte del pretendido manuscrito de Arlt resulta sospechosa: en efecto, la mayoría de los personajes arltianos proviene de la clase baja de los "sirvientes" (107), pero evidentemente se trata de una alusión directa a un ensayo de Jaime Giordano, "Roberto Arlt o la metafísica del siervo" (1968). Pero en la descripción de Lettif se introduce, además, otro texto ajeno a Arlt y Piglia: su descripción física coincide sospechosamente con la que nos ofrece Onetti en su semblanza del propio Arlt: "los huesos frontales del genio (Arlt) muestran una protuberancia en el entrecejo" (14); igualmente se lee en "Homenaje" que Lettif tenía "frontales pronunciados ... en el medio de la frente presenta una protuberancia marcadísima" (107).

Como debe haber quedado claro ya a lolargo de estas notas, Piglia-narrador espera de su lector que desempeñe el mismo papel de detective que el Pigliaactor del texto. Acertada y veladamente, hace entrar en juego a Onetti,uno de los escritores rioplatenses más influidos por la escritura de Arlt: sutilmente comenta, con la descripción que ofrece Onetti de Arlt, el carácter evidentemente no "académico" del texto que prologaba Los siete locos en su traducción italiana ${ }^{7}$. En un prólogo , en principio, cabía esperar una introducción bien documentada y explicatoria, sin embargo, Onetti apunta algunas anécdotas, dudosamente "verídicas". El propio Onetti ironiza sobre el típico prólogo académico: "imagino

5 Vanasco, Prólogo a Regreso de Arlt, 18; cf. S. Gostautas, 1977 sobre la influencia de Dostoievski, y Rita Gnutzmann, 1984, 46s.

- El título anotado en la "página más abajo", El criminal en la selva de ladrillos está evidentemente inspirado en una novela prometida por Arlt, El bandido en el bosque de ladrillos, cf. O. Borré, 13.

${ }^{7}$ I sette pazzi. Bompiani, 1971; su versión castellana se public6 en la revista montevideana Marcha, 28 de diciembre de 1971. Piglia da la correcta pista en la nota 13, pero evidentemente a la vez despista al lector con el nombre falso de Kostia. 
y sé la gran carcajada que le provocaría a Roberto Arlt ... Oigo su risa desfachatada, repetida en los últimos años por culpa de exégetas y neodescubridores" (7).

Apuntemos otro detalle onettiano retomado y transformado por Piglia. Ya se ha mencionado que las anécdotas de Onetti sobre Arlt pueden ser "ficciones" del autor, sobre todo su personaje Kostia que reaparece en "Homenaje" y que parece salido de una novela del propio Onetti. Este dice expresamente que el modelo "real" se llamaba Italo Constantini, pero Piglia le da otro nombre falso, "Saúl" Kostia.

Resulta evidente que el propio texto de Piglia practica lo que atribuye a la pluma de Arlt: la literatura como robo y falsificación, pero no sólo al nivel de la historia, sino también al nivel del discurso: un texto que a veces es y no es un cuento policiaco y un estudio filológico y editorial.

Esta pretendida labor filológica se apoya, además, en varios elementos "científicos":

-la descripción del manuscrito ("Las notas continúan en la página siguiente"), -fija el texto con todas sus variantes (cf. nota 10),

-introduce notas aclaratorias a pie de página,

-subdivide el material en partes (se menciona la "II Parte y III Parte"),

-informa sobre omisiones por dificultad de restablecer el manuscrito ("siguen varios párrafos ilegibles", 114),

-reconstruye el texto en pasajes de lectura dudosa (nota 5, nota 6).

Las diferentes lecturas que el investigador Piglia pretende establecer no son inocentes a pesar de las apariencias, sino que interpretan los textos arltianos, donde en efecto se dan la mano el "cínico" y el "científico" (nota 6) como es el caso de la mayoría de los personajes masculinos y en especial el de Erdosain. La "nota 5" en la que se confunden "descubrir" y "describir", se convierte en una pista para el lector-detective de "Homenaje", igual que las afirmaciones, bien claras, de la página 136: "Me sentía como el detective de una novela policial" y la nota 17 :

Un crítico literario es siempre, de algun modo, un detective ... A la vez, esta asimilación ... de la crítica con la persecución policial, está presente con toda nitidez en Arlt ... (136).

Lo correcto de esta afirmación en el caso de la escritura arltiana es evidente, pero lo es incluso más para el propio Piglia, como muestra el texto objeto de este análisis, y la novela posterior, Respiración artificial. También resulta sospechoso el hecho de que no transcriba todo el material que ha encontrado, sino que salte algunos capítulos, como cuando dice "Tres o cuatro capítulos más adelante ...". Como bien ha visto Gérard Genette, ningún resumen ( $\mathrm{y}$ aún menos una eliminación) es inocente: "ninguna reducción ... puede ser transparente, insignificante, inocente: dime cómo resumes y te diré cómo interpretas" (316). 
Lo que evidentemente tenemos delante es una interpretación de la obra arltiana y una imitación "a la manera de" su modelo. En esta imitación que constituyen las falsas notas arltianas en letra pequeña (104 a 125) introduce temas que el lector arltiano reconoce en seguida: la presencia de un padre tiránico, la humillación del hijo, la posible relación perversa de un hombre con una niña, las pretensiones científicas de los personajes masculinos, elementos folletinescos (aqur la gitana), el asesinato de la mujer (prácticamente copiado del de la Bizca), la argumentación filosófica (en la línea nietzscheana), el modelo literario (Dostoievski), la convivencia con una prostituta "pura", la lucha revolucionaria, la supervivencia mediante la explotación de prostibulos, aparte de otros elementos mencionados anteriormente.

Pero incluso imita y comenta indirectamente recursos técnicos: en efecto, la novela maestra de Arlt constituye un "relato posterior" de Erdosain a su "cronista" ("Homenaje" 110) y la mayoria de sus personajes o son escritores fracasados o por lo menos llevan un diario como Lettif ${ }^{8}$ y son asiduos lectores como éste. Piglia finge ofrecer una muestra de las lecturas heterogéneas de Arlt en la p. 119s.: libros sobre química y física, novelas, textos sobre el anarquismo, todo mezclado con las anotaciones del dinero que debe. Tal vez debería haber incluido algún folletín entre los textos citados. Pero también están presentes otros rasgos arltianos: la dispersión, la combinación y la yuxtaposición de elementos dispares. Por ejemplo, se interrumpen las anotaciones sobre el anarquismo (114)con una "carta de amor", cursi y pretenciosamente "cientifica", pero por la misma razón perfectamente imaginable en una novela arltiana: " $\mathrm{Si}$ no venís me mato. Me dispararé un poco más arriba del corazón ...”.

La proliferación y dispersión (o "máquina polifacética", "Homenaje" 100; Respiración 170) de los textos arltianos es comentada indirectamente en la página 116, aunque está presente en todo el texto: al comienzo se nos promete el cuento "Luba", luego se introducen las notas para una novela sin título; al parecer, en la página 116, se resume el cuento prometido ("Un tema: el hombre puro y la mala mujer en una situación extrema. Encerrados ..."), pero de repente se introducen nuevos materiales para aguafuertes sobre Gorki y Lenin, los cuales a su vez dan paso a notas sobre el anarquista Radowitski, para aludir de nuevo al cuento "Luba" y así sucesivamente durante tres páginas más.

Para terminar el análisis de esta (extensa) parte pseudo-filológica volvamos al nombre de Borges, mencionado antes de paso. El propio Piglia cita el cuento "Pierre Menard, autor del Quijote" de este escritor para enlazarlo con otro de Arlt, "Escritor fracasado": los dos, según la interpretación de Piglia, tienen como tema la idea del escritor como ladrón y falsificador (132). Pero, más que esta comparación de los dos autores (en Respiración Piglia muestra que "El indigno" de Borges es una transposición de la novela El juguete rabioso, 172),

${ }^{8}$ Adan Hayes enfoca todo su estudio Roberto Arlt: la estrategia de su ficción desde el aspecto del fracaso y de la falsificación literaria de los personajes-narradores. 
nos hace pensar en Borges su manía de comprobar la veracidad de sus textos con otros libros, manuscritos y bibliotecas reales con los cuales mezcla hábilmente inventos suyos. Ya vimos que títulos de la bibliografía arltiana existentes se confunden con otros inventados, igual que los nombres verdaderos son falsificados a medias ("Saúl", Kostia). Una de las revistas más conocidas en Argentina es la mencionada Claridad; Piglia pretende que en el no. 24 de agosto de 1941 se publicó un poema de Kostia. Como en el caso de las falsas pistas borgianas, sería imposible encontrar ese número, puesto que el ejemplar de enero de 1931 que incluye un capítulo de Los lanzallamas ya lleva el No. 222 (100 según la nueva serie) $)^{9}$. También el pueblo donde reside Kostia resulta un guiño al lector, ya que se sabe que Borges pasó su infancia en Adrogué, como recuerda con nostalgia en el poema del mismo título de la antología El hacedor (1960). Pero tampoco es imposible que se trate de una autorreferencia, puesto que el mismo Piglia nació en Adrogué y el viaje de Arlt al pueblo para ver a Kostia sería la visita(ción) del "precursor" a su discípulo (cf. "nota $18^{\prime}$ ).

Todos estos datos hacen pensar que Piglia o bien ya estaba trabajando en su novela Respiración cuando escribió "Homenaje" o bien volvió sobre el viejo texto para reelaborarlo posteriormente. La segunda parte de Respiración se centra en una revisión de la literatura argentina en la que se opone como dos polos a Arlt y Borges. A pesar de que Piglia, en la discusión ficticia, parezca inclinarse exclusivamente por la escritura del primero, resulta obvio que él mismo utiliza muchos recursos borgianos. Además tiene en común con Borges el empleo del cuento policíaco tras el cual se esconde una preocupación o bien metafísica o bien metaliteraria. La novela Respiración elabora al máximo esta bipolaridad: por un lado es ficción (histórica) y por otro lado es metanarración.

Volvamos al cuento "Homenaje". El final de la parte 2 cambia de nuevo el tipo de discurso, del erudito filológico al ficticio. En la parte 3 se desarrolla el encuentro entre el detective Piglia y el supuesto ladrón Kostia, pero que no excluye el discurso metaliterario, ya que Kostia tiene "como todos en este país, una teoría sobre Roberto Arlt": evidentemente expresa las ideas de su propio demiurgo sobre la literatura como plagio y falsificación (133). La persecución del manuscrito se complica y lleva a un primer clímax falso, con la publicación del cuento de Arlt firmado con el nombre de Kostia bajo el título Nombre falso: $\mathrm{Luba}$. Con ello el narrador introduce nuevamente falsas pistas; primero porque sabemos que el libro de Piglia del que forma parte "Homenaje" se titula Nombre falso. ¿Quiere sugerir una doble falsificación: por parte de Kostia del material de Arlt y por parte del narrador Piglia del título del texto de éste? La otra pista (o des-piste) es la alusión a la relación de Kafka con Max Brod, al que aquél pidió

\footnotetext{
9 Piglia resume parte de la escritura de Borges en Respiracion de esta forma: "la ficción de Borges: textos que son cadenas de citas fraguadas, apocrifas, falsas, desviadas ... exaspera y lleva al límite, clausura por medio de la parodia, la línea de la erudición cosmopolita y fraudulenta" (162s).
} 
que destruyera toda su obra tras su muerte. Brod evidentemente no cumplió la voluntad de su amigo y se insinúa que podría haber publicado las narraciones kafkianas bajo su propio nombre, tal como lo hace Kostia ${ }^{10}$. De nuevo nos encontramos con la idea de la literatura como mercancía y falsificación. El narrador finge discutir "tres hipótesis". En realidad "falsifica", porque ofrece cuatro (la última escondida en la nota 18) de por qué no se publicó el cuento de Arlt antes. Astutamente nos "roba" una quinta posibilidad: la de que el cuento no sea de Arlt sino del propio Piglia". Las alusiones a la "fidelidad" de Brod pretenden despistar al lector: no se refieren a la "fidelidad" de Piglia para con el texto de Arlt (Kostia ya lo denunció como egoísta: "Usted quiere usar este relato para hacer méritos" ${ }^{n}$ 137) sino al contrario: Piglia adjudica un texto suyo a su maestro. No carece de ironía su juicio sobre su propio cuento al calificarlo como "entre los mejores relatos" (142).

En un súbito giro, típico del relato detectivesco, el narrador saca de su sombrero mágico la solución feliz: en la parte 4 aparece el deus ex machina, Martina, con la caja de manuscritos de Arlt, que contiene el auténtico cuento "Luba", el cual se imprime como apéndice.

¿Con qué datos cuenta el crítico para estar seguro de que, en efecto, "Luba" es un cuento de Piglia y no de Arlt? (aparte de una conversación personal con Piglia en un congreso en Nueva York en junio de 1987). Ya se han mencionado algunos: el personaje de Kostia (el mismo personaje marginado podría salir tanto de una novela de Arlt como de una obra onettiana), las citas de Onetti, las falsas pistas de textos apócrifos, etc. También llama la atención el papel esencial que desempeña un objeto, la caja, en "Homenaje" y en "La caja de vidrio". En el primer caso se insiste, sin ninguna necesidad, en que se trata de una "caja de metal". Pero avanzamos en terreno más seguro si comparamos línea por línea los dos comienzos de "Luba" incluidos en "Homenaje" y constatamos, sin sorpresa, que de nuevo ha ocurrido un robo: en la página 118 falta claramente la frase "La casa era de las de primera clase" (aparte de la palabra "baja" que figura en la p. 147). No sin ironía, el "filólogo" Piglia nos remite en este mismo lugar a una nota a pie de página para explicar que, en la copia mecanografiada del manuscrito, falta un párrafo; otra pista para despistar al lector.

Pero queda todavía otro dato llamativo en el texto. Piglia afirma en el primer párrafo de "Homenaje" que "está en juego la propiedad de un texto de

${ }^{10}$ Kafka desempeña también un papel importante en Respiración, donde se relata su encuentro - fantasioso- con Hitler. Pero sirve sobre todo para hacer una velada descripción de la Argentina de los coroneles: "un mundo donde todos pueden ser acusados y culpables, la siniestra inseguridad que el totalitarismo insinúa en la vida de los hombres, el aburrimiento sin rostro de los asesinos, el sadismo furtivo", 265.

"11 Aden W. Hayes, "La revolución y el prostíbulo: 'Luba' de Roberto Arlt", interpreta el cuento como si fuera un auténtico inédito de Arlt, prueba de hasta qué punto Piglia ha conseguido su "falsificación". 
Roberto Arlt... El texto se llama Luba". Después de seguir varias pistas y comprobar la existencia del cuento buscado, al final, el narrador parece haber establecido la autoria del cuento. Pero resulta sospechoso que en otro momento, también en el contexto de "Luba", menciona otro título: "La propiedad es un robo ${ }^{n}(118)$. Parece lógico relacionar la frase con las ideas expresadas por Piglia con respecto a los cuentos "Escritor fracasado" y "Pierre Menard": la literatura no como invento original, sino como copia, falsificación y robo. El verdadero tema de "Homenaje", por lo tanto, no es la "propiedad arltiana" de "Luba", sino la impropiedad de que un texto tenga un autor único. Piglia lleva la idea de la intertextualidad mucho más allá de las pequeñas coincidencias e influencias: cada texto es la reescritura de uno o muchos textos anteriores. Recuerda la idea borgiana de un supertexto en el cual participan todos los textos. Borges ve relaciones y repeticiones en los textos más dispares a lo largo de los siglos y, en "Tlön, Uqbar, Orbis Tertius", afirma: "se ha establecido que todas las obras son obra de un solo autor, que es intemporal y es anónimo" (Ficciones 27). Por ello, los escritores en Thön no firman sus libros y no existe palabra para lo que denominamos plagio. En Otras inquisiciones, Borges afirma que Valéry piensa que la historia de la literatura es la historia de un espíritu productor: "Esa historia podría llevarse a término sin mencionar un solo escritor" (17).

"Homenaje" tiene en común con muchos relatos de Borges la inclusión explícita de ideas acerca de la forma de contar. Ambos, además, mezclan los géneros: combinan el ensayo con el cuento. Para "Homenaje" hemos comprobado cierta alternancia, pero también la yuxtaposición de la ficción con la monografía literaria con la parafernalia de notas, referencias bibliográficas, citas, etc. ${ }^{12}$. Lo mismo se observa en textos borgianos como "Pierre Menard", "Examen de la obra de Herbert Quain", "La busca de Averroes" etc. "Homenaje a Roberto Arlt" podría llamarse igualmente "Homenaje a Jorge Luis Borges": usa la idea arl tiana del texto como mercancía y la borgiana de un gran intertexto. Practica la mezcla de géneros al estilo de Arlt -quien incluye fórmulas físicas y matemáticas, croquis, etc.- pero igualmente al estilo de Borges, inventor del cuento-ensayo de contenido filosófico y literario.

Por último, Borges fue uno de los primeros escritores en preocuparse del papel del lector en la obra literaria, como muestran los textos "Kafka y sus precursores" y "La obra de Herbert Quain", entre otros. Al final de este cuento se dice explícitamente que el lector debe buscar la verdadera solución que el detective no encuentra. Lo mismo ocurre en "Homenaje": el lector a lo largo del texto persigue pistas como el detective-narrador Piglia, pero lo debe superar, ya que éste lo engaña con la falsa solución de un cuento arltiano. Podemos aplicar

\footnotetext{
12 Es curioso observar que casi simultáneamente con "Homenaje" se publica un libro muy parecido, La orquesta de cristal de Enrique Lihn, Buenos Aires: Sudamericana, 1976, que practica la misma mezcla de estudio literario con ficción.
} 
perfectamente la frase de "Herbert Quain" al lector de "Homenaje": "El lector de este libro singular es más perspicaz que el detective" (Obras 462) ${ }^{13}$.

\section{BIBLIOGRAFIA}

Arlt, Mirta y Omar Borré. Para leer a Roberto Arlt. Buenos Aires: Torres Agüero, 1984.

Arlt. Roberto. Los siete locos. Buenos Aires: Fabril, 1968. Los lanzallamas. Buenos Aires: Fabril, 1968.

Obra completa. Buenos Aires: Carlos Lohlé, 1981. Aguafuertes porteñas. Buenos Aires: Editorial Futuro, 1950.

Aguafuertes porteñas. Buenos Aires: Losada, 1973.

Cronicón de sí mismo. Buenos Aires: Edicom, 1969.

Nuevas aguafuertes porteñas. Buenos Aires: Hachette, 1960.

Borges, Jorge Luis. Ficciones. Buenos Aires: Emecé, 1966.

Otras inquisiciones. Madrid: Alianza-Emecé, 1981.

Obras. Buenos Aires: Emecé, 1974.

Borré, Omar, Prólogo a Roberto Arlt. Estoy cargada de muerte. Buenos

Aires: Torres Agüero, 1984.

Bosquet, Alain. "Une œuvre vénéneuse et lyrique”. Magazine Littéraire, 1981, 61.

Costa, Marithelma. "Entrevista con Ricardo Piglia". Hispamérica, 44, 1986, 3954.

Deredita, John F. “¿Es propiedad? Indeterminación genérica, intertextualidad, diseminación en un texto 'de' Ricardo Piglia" en Texto/Contexto en la literatura Iberoamericana. Actas del XIX Congreso del Instituto Internacional de Literatura Iberoamericana, Madrid. 1980, 61-69.

Genette, Gérard. Palimpsestos. Madrid: Taurus, 1989.

Giordano, Jaime. "Roberto Arlt o la metafísica del siervo". Atenea, CLXVI, 419, enero-marzo 1968, 73-104.

Gnutzmann, Rita. RobertoArlto el arte del caleidoscopio. Bilbao: Univ. del País Vasco, 1984.

"Bibliografía selectiva sobre Roberto Arlt". Revista Interamericana de Bibliografía, xxxix,1, 1989, 3-20.

"Historiay metaficción en Respiración artificial de RicardoPiglia". Revista Interamericana de Bibliografía, vol.XL, no.3, 1990, 351-60.

Goloboff, Gerardo Mario. "La primera novela de Roberto Arlt: el asalto a la literatura". Revista de crítica literaria latinoamericana, 2, 1975, 35-49. Genio y figura de Roberto Arlt. Buenos Aires: Eudeba, 1988.

\footnotetext{
${ }^{13}$ Después de terminado este estudio, he encontrado el breve, pero excelente trabajo de John F. Deredita (cf. bibliografía). Existen lógicamente, algunas coincidencias.
} 
Gostautas, Stasys. Buenos Aires y Arlt. Dostoeivski, Martínez Estrada y Escalabrini (sic) Ortiz. Madrid: Insula, 1977.

Guerrero, Diana. Roberto Arlt, el habitante solitario. Buenos Aires: Granica Editor, 1972.

Hayes, Aden W. Roberto Arlt: la estrategia de su ficción. London: Tamesis, 1981.

"La revolución y el prostíbulo: "Luba" de Roberto Arlt". Ideologies and Literature, II,1, Spring 1987, 141-147.

Jitrik, Noé. "Entre el dinero y el ser (lectura de $E l$ juguete rabioso de Roberto Arlt)". Dispositio, I,2, 1976, 100-133.

Larra, Raúl. Roberto Arlt el torturado. Buenos Aires: Quetzal, 1962.

Morello-Frosch, Marta. "Significación e historia en Respiración artificial de Ricardo Piglia", en Fascismo y experiencia literaria: Reflexiones para una recanonización. Ed. Hernán Vidal. Minneapolis: Ideologies and Literature, $1985,489-500$.

Onetti, Juan Carlos, Prólogo a Roberto Arlt, El juguete rabioso. Barcelona: Bruguera-Alfaguara, 1979, 7-16.

Piglia, Ricardo. "Roberto Arlt: una crítica de la economía literaria". Los libros, 29, marzo-abril 1973, 22-27.

"Roberto Arlt: la fícción del dinero". Hispamérica, 7, 1974, 25-28. Nombre falso. Buenos Aires: Siglo XXI, 1975. Respiración artificial. Buenos Aires: Pomaire, 1980.

Scroggins, Daniel C. Las aguafuertes porteñas de Roberto Arlt. Buenos Aires: Ediciones Culturales Argentinas, 1981.

Vanasco, Alberto, Prólogo a Roberto Arlt, Regreso. Buenos Aires: Corregidor, 1972. 